\title{
L'éducation au handicap en contexte scolaire : une approche par les représentations sociales dans une population lycéenne
}

\section{Education to disability in a school environment: an approach by social} representations within a high school student group

\author{
Maryse Cadet-Mieze ${ }^{1}$ \\ ${ }^{1}$ Laboratoire ADEF, Aix-Marseille Université, France, maryse.cadet-mieze@wanadoo.fr
}

RÉSUMÉ. La démarche d'inclusion et de transformation des représentations sociales du handicap attribuée à l'école laisse place à l'émergence de dynamiques éducatives pour aborder un enjeu sociétal du 21ème siècle : le "vivre ensemble ». La rencontre avec l'autre différent n'est pas un « allant de soi » (Jodelet, 2003). Les représentations sociales du handicap sont le plus souvent entachées d'une vision subjective stigmatisante et restrictive qui fait obstacle au "vivre ensemble ». La théorie des représentations sociales est utilisée dans cette étude comme un outil à l'usage d'une réflexion sur les éducations à. Nous interrogeons le vécu et l'information à l'école sur le handicap comme facteurs potentiels de transformation des représentations sociales des lycéens dits ordinaires. Sur la base de questionnaires, nous définissons une cartographie représentationnelle du handicap d'un groupe de lycéens. Puis, nous conduisons une expérimentation sur deux années en classe de travaux personnels encadrés. L'expérimentation tend à montrer que, par l'approche des éducations à, un vécu avec le handicap est possible à l'école, et qu'il est facteur potentiel de transformation positive des représentations sociales. Mais les obstacles au « vivre ensemble » demeurent.

ABSTRACT. The inclusive and transformative approach of social representations imputed to the School provides a spot for uprising teaching dynamics to address a social issue of the XXIst century: coexistence. The encounter with the Other isn't "self-evident" (Jodelet, 2003). Social role conceptions of disability are often tainted with a subjective stigmatizing and limited vision that creates a barrier to "coexistence". The social role conceptions theory is used within this thesis as a tool for thinking on "educations to". We will question the experience and the School's information on disabilities as potential factors of transformation of the social role conceptions of said "ordinary" high school students. Based on surveys, we will define a representative mapping of disability in the high school students group. Then, we will lead an experiment within two years in the "Travaux Personnels Encadrés" (TPE, stands for Personal Framed Activities) class. The experience tends to show that - with the teaching approach of "educations to" an experience with disability is possible within the School, and that it is a potential factor of positive transformation of social role conceptions. However, the barriers to "coexistence" are still standing.

MOTS-CLÉS. handicap, éducations à, représentations sociales, expérience vécue, vivre ensemble.

KEYWORDS. disability, education to, social representations (social role conception), experience, coexistence (living together).

\section{Introduction}

Le «vivre ensemble» s'entend comme un enjeu démocratique pour l'école d'aujourd'hui et de demain. Mais les représentations essentialistes du handicap (Martinez \& Gadchaux, 2014) fait obstacle à la compréhension et à la rencontre. Elle s'appuie sur un débat qui oppose plutôt qu'il ne rassemble. La loi Handicap ${ }^{1}$ instaure une approche biopsychosociale du handicap mettant en exergue le rôle de l'environnement. Le handicap est à décrypter à partir des capacités de la personne et de l'environnement dans lequel elle se situe, et non sur les seules déficiences. L'environnement doit rassembler. Mais plus de dix ans après la promulgation de la loi, les pratiques de référence ne répondent pas en tous points aux mesures préconisées.

\footnotetext{
${ }^{1}$ Loi n ${ }^{\circ}$ 2005-102 du 11 février 2005 «pour l'égalité des droits et des chances, la participation et la citoyenneté des personnes handicapées », JORF $n^{\circ} 36,2353$. 
Nous usons du mot handicap au singulier approchant, comme d'autres auteurs avant nous (Ameisen, Heilbrunn, Héritier \& Hunyadi, 2010), le handicap particulier vécu à travers différentes appréhensions plutôt qu'à partir de ces différents typologies notamment définies par la loi de 2005. L'étymologie du mot «handicap» est oubliée. Dans l'expression anglophone « hand in cap $»^{2}$, le rééquilibrage des chances est recherché ; le plus fort supportant un désavantage supplémentaire pour compenser son avantage. Mais l'approche commune du handicap inverse le sens originel ; le handicap apparait comme une caractéristique rattachée à la personne d'une infériorité à supporter. Il n'est dès lors plus question de demander à l'individu qui a la chance de n'avoir ni déficience, ni incapacité, ni désavantage de compenser. C'est à la personne handicapée de s'adapter à l'environnement, ce qui va à contre-courant de l'approche retenue par la loi Handicap.

Le législateur confie à l'école inclusive une responsabilité sociétale, celle de transformer positivement les représentations sociales (RS) du handicap pour accompagner l'évolution des pratiques. L'école ouvre ses portes à l'élève différent. Mais qu'en est-il de l'accompagnement éducatif des élèves dits ordinaires dans une visée transformatrice de leur RS du handicap ? La présence du camarade différent suffit-elle en soi ? A côté du dispositif de scolarisation en milieu ordinaire de l'élève différent, l'école a le rôle d'éduquer à la différence. Il est question de contribuer à transformer les représentations en développant les attitudes positives et le respect face à la différence (Harma \& Gombert, 2011).

Mais comment éduquer à une différence dans la visée d'un «vivre ensemble » qui n'est pas un « allant de soi » (Jodelet, 2003) ? Le législateur ne donne pas le mode d'emploi. Dès lors, la recherche en éducation est sollicitée pour penser une éducation au handicap.

La démarche vers la rencontre de l'autre différent se doit d'être accompagnée. Harma et al. (2014) relèvent qu'il serait bien naïf de croire que la seule présence du camarade différent dans la classe suffise pour conduire à un changement des attitudes. Nous pouvons penser deux approches éducatives :

- la création d'un environnement favorable à l'évolution des attitudes et des comportements (Belmont \& Vérillon, 2003, Harma \& Gombert., 2011, Rousseau \& Belanger, 2004). Un environnement «propice », comme le dispositif de scolarisation en milieu ordinaire, aiderait les élèves dits différents et aurait des répercussions sur les comportements des élèves dits ordinaires (Harma et al., 2013). Mais le comportement ne renseigne pas sur les RS. Il peut n'être qu'une réponse à la commande sociale.

- des programmes ou actions visant à dé stigmatiser le handicap (Harma et al., 2014). Le handicap est le plus souvent abordé à l'école à la seule initiative de l'enseignant ou du corps enseignant d'un établissement. Des actions de sensibilisation dans la classe où l'établissement reçoit des collectifs d'associations, l'organisation de sorties, parfois la réalisation de projet, sont conduites. La difficulté de telles initiatives est d'en comprendre et d'en cerner les contours et les finalités, pour l'enseignant, pour les intervenants (souvent extérieurs) et pour les élèves. Nous posons la question d'un effet durable des actions de sensibilisation.

L'émergence des éducations à offre une gamme de créativité et de diversité pour penser l'approche de la question du handicap à l'école. Le cadre conceptuel des éducations à permet une approche pluridisciplinaire de l'objet social et une implication de l'élève qui paraissent indispensables pour répondre à la finalité transformatrice de la démarche éducative.

Une expérimentation sur deux années au lycée exploite l'outil $\mathrm{TPE}^{3}$ en y incluant une double dimension relationnelle et réflexive pour une dé contextualisation et re contextualisation de l'objet handicap par les lycéens.

\footnotetext{
${ }^{2}$ La main dans le chapeau, issu d'un jeu hippique irlandais ou d'un jeu de hasard anglais.

${ }^{3}$ Travaux personnels encadrés
} 
La technique de l'analyse du discours (Bardin, 1977, 2011) permet d'identifier chez le sujet-élève la chronologie de l'approche de l'objet, l'implication et l'engagement, l'ancrage et les connaissances acquises.

Les dimensions relationnelle et réflexive sont sources de vécu et d'information sur l'objet. Les enseignements retirés de la théorie structurale des RS (Abric, 1994) laissent penser les éléments de vécu et d'information comme facteurs potentiels de transformation des RS des lycéens dits ordinaires.

Le présent article présente une recherche sur un objet prioritaire sur le plan sociétal (le handicap) et la possibilité de recourir à l'éducation à pour transformer les RS des lycéens sur l'objet.

\section{Cadre théorique}

Les modèles et outils théoriques choisis sont la théorie structurale des représentations sociales, (Abric, 1994 ; Jodelet \& Moscovici, 1989; Moscovici, 1961 ; Rouquette \& Rateau, 1998) et le cadre conceptuel des éducations à (Barthes \& Alpe ; 2012, 2016 ; Beitone \& Legardez, 1997 : Floro, 2013 ; Garnier \& Sauvé, 1999 ; Lange \& Barroca-Paccard, 2017 ; Legardez \& Simmoneaux 2011 ).

\subsection{L'utilisation de la théorie des représentations sociales}

La recherche en éducation s'intéressent aux aspects caractéristiques du processus des RS et à l'utilisation d'outils d'analyse des RS (Barthes \& Alpes, 2016; Garnier \& Sauvé, 1999; Legardez, 2004 ; Poplimont, 2003).

Moscovici (1961) définit deux processus fondamentaux : l'objectivation et l'ancrage. Comme le soulignent Jodelet et Moscovici, l'objectivation est le processus par lequel le sujet sélectionne des éléments d'un objet pour construire un schéma organisationnel qui modèle l'objet en image concrète et préhensible au sein du groupe d'appartenance (1989). L'ancrage est le processus par lequel l'objet s'enracine dans des RS constituées de savoirs antérieurs, de significations propres au groupe d'appartenance du sujet partageant les mêmes valeurs sociales (Ibid). L'ancrage explique les pratiques sociales communes sur l'objet de RS. Les processus de RS d'un objet tendent au décodage et à la lecture structurée et organisée d'une réalité projetée. En cela, ils allouent aux RS une fonction d'orientation de la communication sociale et des pratiques sociales autour de l'objet. L'étude des RS conduit également à approcher la dynamique des relations sociales autour de l'objet, et des rapports du sujet-élève ou groupe-élèves à l'objet et de là les conflits internes et externes.

La théorie des RS, utilisées pour aborder la question de l'éducation au handicap à l'école, permet d'approcher l'objectivation et l'ancrage. Cela tend à la compréhension des dynamiques conduisant à une prise de position, à un comportement, à la relation avec l'objet. Cette compréhension est susceptible d'aider à penser une éducation au handicap dans l'objectif du " vivre ensemble ». Garnier et Sauvé (1999) soulignent la pertinence «d'ancrer l'apprentissage dans les éléments de représentations initiales, pour ensuite enrichir le champ représentationnel vers une prise en compte de la pluralité des modes de relation à l'environnement » (p. 72).

Les représentations comportent des éléments socialement construits et partagés au sein d'un groupe d'appartenance (Moliner, Rateau \& Cohen-Scali, 2002). Mais les représentations se composent aussi d'éléments idiosyncrasiques liés à sa propre histoire, à son vécu, indissociables de son environnement idéologique et social. Aussi les RS sont-elles porteuses de différences individuelles. La complexité des RS tient du fait que nous y retrouvons une logique particulière construite sur des contradictions, des dissonances. Le vécu est aussi individuel et porteur de signification. Dès lors comment l'école peut-elle travailler avec et sur le vécu, facteur potentiel de transformation? 


\subsection{L’approche éducative par les éducations à}

A travers la théorie des représentations sociales en éducation, nous faisons le choix d'un paradigme de recherche fait autant pour l'analyse que pour l'agir qui vise à transformer les pratiques et la réalité sociale. Si les RS sur le handicap des lycéens dits ordinaires est obstacle au « vivre ensemble », l'éducation à reste possible et la théorie structurale des RS permet de manifester un changement. Dès lors, concevoir un agir pour la formation et la transformation, pour assumer le changement par l'éducation à devient perceptible par des indicateurs dans les paroles, à travers une démarche empirique.

Pour Barthes et Alpe (2012), la perception plus grande des problèmes sociaux conduit dès les années 1990 en France à l'émergence d'une éducation à, avec notamment les recherches en éducation au développement durable (Girault \& Sauvé, 2008), en éducation civique juridique et sociale (Alpe \& Legardez, 2000 ; Legardez \& Simmoneaux, 2011), ou encore en éducation à la santé (Eymard, 2004). La recherche interroge notamment le partage entre l'instruction basée sur les savoirs et l'éducation basée sur les valeurs (Audigier \& Tutiaux-Guillon ; 2008, Barthes \& Alpe, 2012).

L'éducation à ne répond pas aux caractéristiques d'une discipline scolaire telles que définies par Audigier (1993) à savoir par ses trois éléments fondamentaux : « un ensemble de connaissances "admises par tout le monde, à tout le moins par les enseignants...", des exercices types fortement identifiés à la discipline et des procédures d'évaluation » (Barthes \& Alpe, 2012, p.2).

Les éducations à répondent à des caractéristiques spécifiques présentées notamment dans le programme de recherche Education au développement durable ${ }^{4}$ :

[...] : une interconnexion des problématiques tant du point de vue éducatif (centration sur des expériences à vivre, des compétences, nature mixte, controversée, des savoirs impliqués) que du point de vue sociétal (imbrication des questions environnementales, sanitaires, sociales et économiques) ; une ressemblance des démarches pédagogiques favorisant leur aspect éducatif et non leur assujettissement à des visées utilitaristes ; la place déterminante et impliquée du sujet dans sa dimension sociale, tant l'élève que l'enseignant, de ses valeurs et de ses systèmes de représentations-connaissances. (Ibid, p. 8)

L'interconnexion éducative et sociétale des problématiques abordées prend en compte les expériences vécues au même titre que les savoirs convoqués. Ce sont tant des savoirs de référence savants (formels) que sociaux (informels), des croyances et des pratiques sociales (non formelles) dans la complexité des imbrications des questions en lien avec le handicap qui touchent les domaines de la santé, du social, de l'environnement, de l'économie, de la cité. La priorité donnée à l'éducation plaçant le sujet-élève au cœur de la démarche pédagogique fait de l'élève un acteur social de sa propre démarche vers un savoir-être, avant un savoir-faire.

Une telle démarche tient compte des systèmes de représentations du sujet-élève. Les éducations à sont à la fois thématiques (et non disciplinaires), en relation étroite avec les questions socialement vives (Legardez \& Simmoneaux, 2006) et en lien avec les valeurs. L'éducation ne peut en rester à l'étape des transformations des représentations mais doit aboutir à une transformation des actions. La réflexion qui en naît doit aider l'élève à devenir un citoyen responsable capable de choix, d'engagement, dans un esprit critique et éclairé sur les valeurs républicaines, telles la responsabilité citoyenne et la solidarité individuelles et collectives, et sur les pratiques et comportements, le tout dans une cohérence de l'environnement social. Un mieux « vivre ensemble » par l'action et par la réflexion tend à l'acceptation de l'autre dans sa différence, à la mixité et à l'enrichissement réciproque des différences de chacun.

\footnotetext{
${ }^{4}$ Programme de recherche ANR EDEAO, Education au développement durable, appuis et obstacles, 2008-2012.
} 


\section{Méthodologie}

Plusieurs outils ont été mobilisés pour cette étude. Nous avons dans un premier temps procédé par une étude «classique » des représentations auprès de lycéens dits ordinaires en lycée technologique et professionnel et en lycée général par voie d'entretiens, questionnaires et techniques d'évocations hiérarchisées qui nous donne une cartographie des RS du handicap de l'échantillon de population. Nous avons ensuite procédé à une étude de cas en lycée général reposant sur une observation directe participante et une analyse du discours d'entretiens semi-directifs en lien avec l'expérimentation menée. L'expérience est conduite en classe de TPE avec un objectif de transformation des représentations et de saisies des données.

Nous avons choisi de conduire notre expérimentation en lycée général pour deux raisons : la première est qu'en lycée général l'objet handicap n'est inscrit dans aucune discipline, ce qui peut être le cas en lycée technologique et professionnel selon la spécialité choisie. La deuxième est l'existence en lycée général de l'outil TPE qui nous apparaît comme un cadre favorable pour aborder une approche éducative par les éducations à.

\subsection{L'utilisation de questionnaires}

Nous avons procédé par questionnaires auprès de 366 sujets avec principalement des questions d'évocations, d'associations de phrases, de substitution (Abric, 1994). L'outil questionnaire permet d'introduire des aspects quantitatifs jugés « fondamentaux dans l'aspect social d'une représentation » (Ibid, p. 62). Il permet par ailleurs une standardisation des questions et des réponses (cf. Tableau 1).

\begin{tabular}{|c|c|c|}
\hline \multirow{2}{*}{ Items } & \multicolumn{2}{|c|}{ Questions principales } \\
\cline { 2 - 3 } & Questionnaire 1 & Questionnaire 2 \\
\hline $\begin{array}{c}\text { les composantes des } \\
\text { RS du handicap } \\
\text { les valeurs et les } \\
\text { normes }\end{array}$ & $\mathrm{n}^{\circ} 2$ à $2-34.5$ & $\mathrm{n}^{\circ} 1,4,5,6 \mathrm{~b}$ \\
\hline $\begin{array}{c}\text { 1'information reçue } \\
\text { sur le handicap } \\
\text { notamment à } \\
\text { l'école }\end{array}$ & $\mathrm{n}^{\circ} 1$ & \\
\hline $\begin{array}{c}\text { le vécu avec le } \\
\text { handicap } \\
\text { notamment à } \\
\text { l'école }\end{array}$ & $\mathrm{n}^{\circ} 3.3,3.4$ & $\mathrm{n}^{\circ} 2,3$ \\
\hline $\begin{array}{c}\text { les sentiments } \\
\text { (ressentis) exprimés } \\
\text { sur l'objet } \\
\text { handicap. }\end{array}$ & $\mathrm{n}^{\circ} 4.1,4.4$ & \\
\hline $\begin{array}{c}\text { Les } \\
\text { positionnements } \\
\text { Les attitudes }\end{array}$ & $\mathrm{n}^{\circ} 2-4,2-5,2-8,6.1$ & \\
\hline $\begin{array}{c}\text { les indicateurs } \\
\text { sociaux }\end{array}$ & $\mathrm{n}^{\circ} 4.2$ & $\mathrm{n}^{\circ} 6 \mathrm{a}, 8$ \\
\hline
\end{tabular}

Tableau 1. Questionnaires 1 et 2 - items

Le premier questionnaire s'articule autour de 6 items sous forme de 6 questions principales : la question 1 sur les normes et valeurs, la question 2 sur les représentations du handicap, la question 3 sur le vécu (expérience, information), la question 4 sur le ressenti et l'attitude, la question 5 sur les indicateurs sociaux, la question 6 sur le positionnement. Chaque question se décomposent en sous questions soit un total de 30 questions, certaines à réponses multiples. Le second questionnaire se compose de 8 principales questions, qui peuvent se décliner soit au total 31 questions (cf. Encadré $\left.\mathrm{n}^{\circ} 1\right)$. 
1- Quels sont les mots ou expressions qui vous viennent à l'esprit quand vous pensez au mot

« handicap »?

2- A quelles occasions avez-vous entendu «parler » du mot « handicap »?

3- Comment avez-vous été sensibilisé au handicap ?

4- Le handicap c'est... ?;

5- Regarder les photos ci-dessous...

6- Pour notre société, le handicap c'est... ?;

7- Qui êtes-vous?

8- Votre positionnement.

Encadré 1. Questionnaire 2 - 8 principales questions

Le deuxième questionnaire débute par une question d'évocation spontanée. Cette question introductive vise à obtenir une réponse spontanée activant le processus représentationnel. Cela permet de repérer les éléments centraux et les éléments périphériques. La question se place en premier afin de ne pas être influencée par les autres questions posées. Les questions 4,5 et 6 répondent au test d'indépendance au contexte (Monaco, Lheureux \& Halimi-Falkowicz, 2008). Le test vise la vérification des éléments centraux et périphériques éventuellement ressortis de la question 1 . La question 4 présente 16 propositions à classer selon une échelle «tout à fait d'accord », «d'accord », «pas d'accord», «pas du tout d'accord». La question 6 recourt à la technique de substitution et repose sur 8 propositions à classer selon la même échelle que la question 4 . La question 5 présente 4 planches photographiques et invite le sujet à choisir celle qui parait la plus représentative du mot «handicap ». Nous cherchons dans les questions 2, 3 et 8 à cerner le positionnement des lycéens sur l'objet du handicap. Les questions 2 et 3 portent sur l'information et la sensibilisation au handicap des lycéens à l'école. La question 8 vise à repérer le positionnement des sujets vis-à-vis du handicap.

Nous faisons le choix de la méthode dite associative dont la visée est de « faire établir (ou à rendre manifeste) un lien entre un inducteur et un induit » (Flament \& Rouquette, 2003, p. 58). Nous utilisons pour nos questionnaires l'association de mot ou expression. Dans le questionnaire 2, nous optons pour le modèle de l'association libre continuée soit plusieurs induits, sans véritable contrainte de production. Nous cherchons à repérer le noyau central des représentations sociales sur le handicap du groupe et à explorer le contenu. La spontanéité de la réponse donnée et la dimension projective doit permettre un accès aux éléments de l'univers sémantique de l'objet étudié.

\subsection{L'utilisation de l'outil TPE}

Le cadre du TPE choisi présente le triple avantage de déjà exister, de conduire à un travail collectif pluridisciplinaire et de se présenter comme un espace propice à la liberté d'expression et à la coréflexion. A son introduction en lycée général, le TPE est présenté comme une étape innovante dans le système éducatif. ${ }^{5}$

Le TPE est une épreuve anticipée obligatoire du baccalauréat introduite dans les classes de première générale. C'est un enseignement distinct des cours traditionnels. Les séances de deux heures consécutives se déroulent sur 18 semaines. Le TPE permet aux élèves de conduire une recherche de manière autonome en vue d'une production écrite (le rapport de TPE) qui est défendue lors d'un exercice oral (la soutenance) devant un jury. Les élèves doivent appuyer leurs travaux sur des recherches documentaires. Le rôle des enseignants et du documentaliste consistent à aider les élèves à trouver des ressources, les diversifier, les sélectionner et les analyser. L'expérimentation menée introduit deux éléments nouveaux: le regroupement des groupes d'élèves par thématique (le handicap) ; la méthodologie de recherche d'investigation sur le terrain.

\footnotetext{
${ }^{5}$ Bulletin officiel nº 26 du 30 juin 2011.
} 
L'expérimentation est menée auprès de 31 lycéens de première en série ES (économique et sociale) et $\mathrm{S}$ (scientifique) ayant préalablement répondu aux questionnaires.

Nous avons regroupé les élèves ayant librement choisi de traiter de l'objet handicap pour leur TPE. Leur choix est orienté par les directives données par l'épreuve et présentées dans les fiches Eduscol : le choix d'un des thèmes nationaux définis en tronc commun et par série et de deux disciplines de référence.

Des groupes de deux à trois élèves se forment soit 11 groupes. Les élèves mènent une investigation pour tenter de répondre à une problématique qu'ils ont préalablement construite et utilisent des outils tels que le questionnaire ou l'entretien. L'expérimentation se veut favoriser les échanges et partages d'expériences développant un conflit sociocognitif : «l'élaboration collective de nouvelles régulations réorganise les régulations individuelles préexistantes » (Doise \& Mugny, 1997, p. 41). Les échanges en classe doivent conduire à la co construction d'un savoir sur l'objet, confrontant savoirs savants, savoirs sociaux et expériences. Avec la recherche sur le terrain, l'élève dépasse les savoirs de référence pour aborder une question de société en se confrontant à la réalité du monde qui l'entoure, en tentant de le comprendre. La méthodologie scientifique permet à la fois une confrontation avec la réalité et une prise de distance. Cela tend à dépasser une réalité non analysée pour rendre compte avec plus d'objectivité de l'objet d'étude: observer un phénomène, tenter de le comprendre, acquérir de nouvelles connaissances, par une analyse rigoureuse et objective des données recueillies.

Les rapports écrits et les entretiens individuels (cf. Encadré 1) sont interrogés sur ce qu'ils peuvent révéler de la construction ou non d'un vécu à travers l'expérience d'une rencontre et d'un travail collectif sur l'objet, et sur le caractère influent ou non en termes de transformation positive du regard porté sur l'objet handicap. Les mêmes grilles de lecture et d'analyse du discours (cf. Tableaux 3 et 4) sont suivies. Les items prédéfinis sont: - la chronologie dans l'approche de l'objet; - l'angle d'approche (notamment par l'utilisation ou non de la méthodologie d'investigation et des outils présentés); - l'implication; - les connaissances retenues ; - la réflexion; - l'engagement; - les difficultés rencontrées ; - les ancrages ; - l'évolution du regard porté sur l'objet.

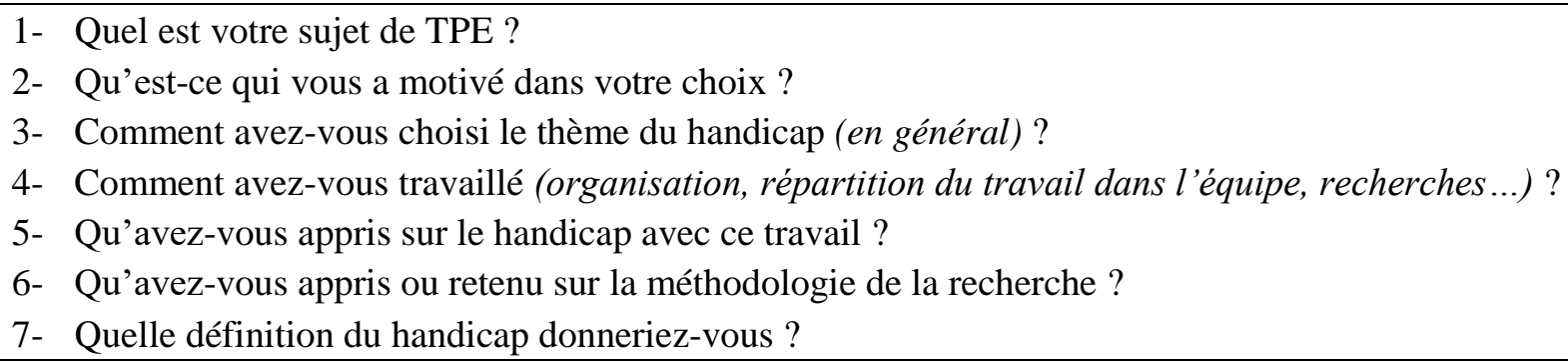

Encadré 2. Grille d'entretien 


\section{Grille de lecture}

La chronologie du choix de l'objet d'étude

L'angle d'approche de l'objet

L'engagement
Choix de l'objet handicap

Choix d'une thématique

Mise en lien de l'objet et de la thématique ou inversement

Institutionnel (référence aux textes)

Recherche (méthodologie de recherche)

Guidance

Réflexion

Implication

Tableau 2. Grille de lecture

\begin{tabular}{|c|c|}
\hline \multicolumn{2}{|l|}{$\begin{array}{l}\text { Discours (écrit/ oral) } \\
\text { Cohérence }\end{array}$} \\
\hline Chronologie & $\begin{array}{l}1^{\text {er }} \text { choix du handicap } \\
1^{\text {er }} \text { choix de la thématique } \\
1^{\text {er }} \text { choix de l'expérimentation } \\
\text { Comment on est entré sur le sujet }\end{array}$ \\
\hline $\begin{array}{l}\text { Démarche directe } \\
\text { Démarche indirecte } \\
\text { Etayage } \\
\text { Pertinence }\end{array}$ & $\begin{array}{l}\text { Implication } \\
\text { Engagement dans l'action } \\
\text { Engagement dans le discours }\end{array}$ \\
\hline Ancrage & $\begin{array}{l}\text { Dans expérience } \\
\text { Vécu (connaissance personne handicapée) } \\
\text { Dans discipline } \\
\text { Vison matérielle } \\
\text { Vision superficielle } \\
\text { Vision rationnelle } \\
\text { Vision institutionnelle } \\
\text { Vision affective } \\
\text { Vision sociale } \\
\text { Vision psycho sociale } \\
\text { Impact de la thématique }\end{array}$ \\
\hline Ce qu'on a retenu & $\begin{array}{l}\text { Des connaissances sur la thématique } \\
\text { Des connaissances sur le handicap } \\
\text { Une réflexion sur la thématique } \\
\text { Une réflexion sur le handicap }\end{array}$ \\
\hline
\end{tabular}

Tableau 3. Grille d'analyse 


\section{Résultats}

Dans un premier temps, la théorie des RS convoquée nous permet de définir une cartographie de des représentations du handicap par l'échantillon de population lycéenne. Elle rend objectivable une transformation dans la tension entre le noyau central et les éléments périphériques observés dans les pratiques qui rendent possibles les évolutions et les attitudes positives. Ce que dans un second temps la démarche praxéologique dans le cadre du TPE tend à démontrer.

\subsection{Une cartographie des représentations sociales du handicap des lycéens dits ordinaires}

Nous avons procédé à une analyse par tri à plat pour dresser un état des lieux du rapport au handicap du groupe lycéen: les ressentis, les positionnements sur certaines questions comme le mode d'information et le vécu à l'école et dans l'entourage. Puis nous avons procédé par tri croisé avec calcul des khi deux pour déterminer l'éventuelle influence de certains éléments entre eux et notamment l'information et le vécu sur le ressenti qui fait obstacle ou favorise le «vivre ensemble ». Enfin, pour approcher la structure des RS du handicap de l'échantillon, nous avons procédé par technique d'association libre et technique de substitution.

Les RS du handicap de l'échantillon de sujets-élèves-dits-ordinaires peut être qualifiée de neutre voire de négative. Elle est restrictive : elle se focalise sur l'aspect visible et physique du handicap et la survenance de caractère accidentel. Comme dans les précédentes études (Harma et al., 2013 ; Morvan, 1988), l'image du «fauteuil roulant» symbolise le handicap physique qui englobe le handicap en général. Aux évocations spontanées, le fauteuil roulant se place en premier rang en termes de fréquence et d'importance. L'image du « fauteuil roulant» donne aux RS du handicap sa signification et sa cohérence (Abric, 1994). Le «fauteuil roulant» en tant que noyau central est lié à la mémoire collective et à l'histoire de la société. Il est homogène, stable, cohérent et rigide dans le groupe. Au « fauteuil roulant» est associé le handicap physique surreprésenté, la difficulté et le besoin d'assistance projetés. La différence est mise en avant pour justifier la position.

Le questionnaire permet de dresser un inventaire des modes d'informations reçues sur la question du handicap par l'échantillon de population. $78 \%$ des lycéens déclarent avoir reçu des informations sur le handicap. Pour la majorité d'entre elles $(74,8 \%)$, la première source d'information est l'école. L'information reçue à l'école prend plusieurs formes qui peuvent se cumuler : les cours magistraux sur l'objet $(48,6 \%)$, les interventions de personnes extérieures $(45 \%)$, les stages $(26,6 \%)$, les projets et exposés $(22 \%)$. En dehors de l'école, sont cités les médias $(47,7 \%)$ et le cadre familial ou relationnel $(32,1 \%)$.

L'information s'avère plus potentiellement porteuse de transformation lorsqu'elle s'accompagne ou se traduit par un vécu avec le handicap. Les sujets déclarent l'école comme première source d'informations sur le handicap, mais ce n'est pas cette information qui paraît significative pour donner au sujet une approche plus «positive » du handicap et écarter des sentiments négatifs (comme le malaise, le rejet) qui font obstacles à la rencontre (cf. Tableau 5). Seule l'information résultant de stages professionnels pour les lycéens en filière spécialisée peut constituer un vécu, facteur potentiel de transformation positive. Le stage permet la mise en lien du savoir et de l'expérience, et la rencontre. En lycée général, l'information susceptible de transformer le regard porté sur le handicap est extrascolaire, via les médias principalement. Nous avons là matière à réflexion pour repenser l'information sur le handicap au lycée. 


\begin{tabular}{|c|c|c|c|c|c|c|}
\hline \multirow{3}{*}{$\begin{array}{l}\text { Informations } \\
\text { reçues }\end{array}$} & \multicolumn{2}{|c|}{$\begin{array}{c}\text { NR } \\
\text { (non réponses) }\end{array}$} & \multicolumn{2}{|c|}{$\begin{array}{c}\text { Oui } \\
\text { Plusieurs réponses } \\
\text { possibles parmi les } \\
\text { propositions }\end{array}$} & \multicolumn{2}{|c|}{$\begin{array}{c}\text { Non } \\
\text { Plusieurs réponses possibles } \\
\text { parmi les propositions }\end{array}$} \\
\hline & Effectif & $\%$ & Effectif & $\%$ & Effectif & $\%$ \\
\hline & 9 & 4,1 & 163 & 78 & 46 & 21,1 \\
\hline $\begin{array}{l}\text { 1- A l'école, } \\
\text { dont : }\end{array}$ & & & 108 & 74.8 & & \\
\hline $\begin{array}{l}\text { Cours } \\
\text { magistraux }\end{array}$ & & & 106 & 48,6 & 112 & 51,4 \\
\hline $\begin{array}{l}\text { Actions de } \\
\text { sensibilisation/ } \\
\text { interventions } \\
\text { de personnes } \\
\text { extérieures }\end{array}$ & & & 98 & 45,0 & 120 & 55,0 \\
\hline $\begin{array}{l}\text { Projets : exposés, } \\
\text { TPE }\end{array}$ & & & 48 & 22,0 & 170 & 78,0 \\
\hline $\begin{array}{l}\text { Apporter des } \\
\text { soins à une } \\
\text { personne } \\
\text { handicapée }\end{array}$ & 57 & 26,1 & 91 & 41.7 & 86 & 39,4 \\
\hline $\begin{array}{l}\text { Stage (cursus } \\
\text { scolaire) }\end{array}$ & & & 58 & 26,6 & 160 & 73,4 \\
\hline $\begin{array}{l}\text { En formation } \\
\text { professionnell } \\
\text { e initiale }\end{array}$ & & & 19 & 8,7 & 199 & 19 \\
\hline $\begin{array}{l}\text { En formation } \\
\text { professionnell } \\
\text { e continue }\end{array}$ & & & 15 & 6,9 & 203 & 93,1 \\
\hline $\begin{array}{l}\text { 2- Dans le cadre } \\
\text { familial ou } \\
\text { relationnel }\end{array}$ & & & 70 & 32,1 & 148 & 67,9 \\
\hline $\begin{array}{l}\text { 3- Par les médias } \\
\text { (TV, radio, } \\
\text { journaux, } \\
\text { internet...) }\end{array}$ & & & 104 & 47,7 & 114 & 52,3 \\
\hline
\end{tabular}

Tableau 4. L'information reçue sur le handicap par les lycéens

\begin{tabular}{|c|c|c|c|c|c|c|}
\hline \multirow[t]{3}{*}{ Malaise } & \multicolumn{6}{|c|}{ Informations reçues } \\
\hline & \multirow{2}{*}{$\begin{array}{l}\text { Informations } \\
\text { en général }\end{array}$} & \multicolumn{2}{|c|}{ Hors Ecole } & \multicolumn{3}{|c|}{ Ecole } \\
\hline & & $\begin{array}{c}\text { Cadre } \\
\text { familial ou } \\
\text { relationnel }\end{array}$ & Médias & $\begin{array}{c}\text { Ecole en } \\
\text { général }\end{array}$ & $\begin{array}{c}\text { Cadre } \\
\text { professionnel } \\
\text { / stage }\end{array}$ & $\begin{array}{c}\text { Actions de } \\
\text { sensibilisation }\end{array}$ \\
\hline $\begin{array}{l}\text { Khideux } \\
\left(X^{2} \text { cal }\right)\end{array}$ & 12,73 & 5,39 & 12,73 & 5,19 & 12,73 & 7,67 \\
\hline $\begin{array}{l}\text { Fisher et } \\
\text { Yates } \\
\left(X^{2} l u\right)\end{array}$ & 9,49 & 9,49 & 9,49 & 9,49 & 9,49 & 9,49 \\
\hline $\begin{array}{l}\text { Cramer } \\
\text { (V) }\end{array}$ & 0,241 & 0,157 & 0,241 & 0,154 & 0,241 & 0,187 \\
\hline Relation & $\mathrm{S}$ & NS & $\mathrm{S}$ & $\mathrm{NS}$ & $\mathrm{S}$ & PS \\
\hline Effectif & 218 & 218 & 218 & 218 & 218 & 218 \\
\hline
\end{tabular}

Tableau 5. Relation malaise / informations 


\subsection{Une étude de cas en classe de TPE}

Nous sélectionnons pour le présent article le travail d'un groupe qui se compose de trois lycéennes en première $\mathrm{S}$, âgées de 16 ans, que nous nommons Charlotte, Lilou et Pétula. Le choix de la présentation d'un seul travail permet une étude plus minutieuse. Nous proposons une lecture des discours extraits du travail collectif écrit et d'un entretien avec l'un des membres du groupe, Charlotte. Le choix de présenter le travail de ce groupe tient à la fois de sa représentativité et de sa richesse qui nous permet d'entrevoir de véritables perspectives éducatives. Le choix porté sur Charlotte s'explique par la personnalité du sujet qui est apparu très vite comme «leader » dans le groupe et le contenu fort riche de l'entretien.

Nous avons choisi de présenter le travail d'un groupe composé uniquement de filles car cette composition se présentait en plus grand nombre - et donc plus représentatif - dans les travaux que nous avons conduits que les groupes composés uniquement de garçons ; nous n'avons pas rencontré de groupes mixtes. Les groupes se sont formés par affinité et par choix pour l'objet à étudier.

Comme pour la majorité des groupes, le groupe choisi se dit plutôt indifférent à la question du handicap au début des séances de TPE mais avoir choisi la thématique par curiosité et pour participer à l'expérimentation. L'expérimentation incluait d'ajouter au travail classique de TPE une initiation à l'investigation de terrain avec l'approche des outils d'enquête comme le questionnaire et l'entretien et la manipulation de logiciels d'analyse de données (excel, dico, Iramuteq).

Le groupe pose la question de la responsabilité de la personne handicapée dans la projection de son image. Le groupe rencontre une étudiante atteinte d'infirmité motrice cérébrale (IMC), que nous nommerons Marie, via des relations communes et l'interviewe une première fois. De retour en classe, le groupe analyse le discours par la technique de la lecture flottante. Des thématiques ressortant de ce travail, le groupe décide de réinterroger Marie. Dès lors, Marie les invite à la suivre dans son quotidien (ses études, ses séances de kinésithérapie, ses cours de natation). C'est par l'analyse des entretiens à travers une lecture flottante, un comptage des mots et une catégorisation par items que le groupe découvre le bienfait du handisport pour Marie et construit sa problématique autour de cette question.

\subsubsection{Un travail collectif : le rapport de TPE}

Le rapport de 37 pages (96 avec les annexes) dépasse largement la consigne donnée de 20 pages. Dès la première phrase d'introduction, le groupe dit sa sensibilisation à la question du handicap « (...) la question du handicap, qui nous intéresse et nous touche tout particulièrement. » (Rapport TPE, p.1). Le groupe dit l'importance de se questionner sur l'objet du handicap : " ce sujet est rarement abordé ; le monde du handicap demeure largement méconnu. » (Rapport TPE, p. 1). Sont énoncés la question de recherche et la problématique: «en quoi le sport est susceptible de valoriser son [la personne handicapée] image sociale? » La méthodologie suivie est présentée : «pour répondre à cette question nous avons décidé de faire une étude de cas. Nous nous sommes donc intéressées au cas de Marie, jeune étudiante souffrant d'IMC (Infirmité Motrice Cérébrale) avec des troubles associés, qui pratique régulièrement la natation depuis son enfance. »(Rapport TPE, p. 2).

Le rapport se divise en trois parties qui correspond aux trois thématiques dégagées par le groupe : la maladie invalidante, la socialisation, la natation. Dans chacune des parties, un paragraphe est consacré à un extrait des entretiens avec Marie. Chaque partie répond au même plan en trois parties : définition, description et contexte ; - théorisation ; - étude de cas (témoignage de Marie).

Le vocabulaire de la recherche est adopté et utilisé. Il est question de rendre compte des données recueillies et de mettre en lien d'autres études consultées. Il est fait application des outils et techniques enseignés comme la question d'énonciation (lorsqu'on vous dit handicap... ?) et d'implication du sujet (pensez-vous que...?) et d'une analyse chiffrée des données (utilisation des logiciels Dico et Iramuteq). 
Une voie est trouvée entre la réponse à la consigne de l'épreuve sanctionnée au baccalauréat et le choix assumé de travailler selon la méthodologie d'investigation, entre la recherche documentaire prescrite et les enquêtes de terrain menées. L'item socialisation est travaillé à partir de la discipline de référence $\left(\mathrm{ECJS}^{6}\right)$ et de lectures. L'item maladie invalidante est travaillé à partir d'une recherche documentaire et d'un entretien avec un médecin. La part créative est pleinement affichée par le choix de traiter autour d'une étude de cas.

Nous proposons une lecture par items suivant la grille élaborée (cf. Tableau 2):

Une rencontre. Le groupe a été gagné par la force de caractère et l'esprit positif du sujet-Marie rencontré et cela se retrouve dans le rapport écrit. Tout le rapport a été monté en fonction des entretiens cliniques.

Une implication. La forte implication du groupe se manifeste par :

- le choix de l'objet d'étude : cela apparaît dès la première phrase de l'introduction : "parmi les thèmes proposés dans le cadre du Travail Personnel Encadré, "Santé et Bien-être » s'est imposé immédiatement à nous, car il nous permettait d'aborder la question du handicap, qui nous intéresse et nous touche tout particulièrement » (Rapport TPE, p. 1) ;

- l'élaboration du travail en grande partie en dehors des séances de TPE, sur le terrain et à la maison : le groupe ne compte pas son temps pour se déplacer sur le terrain, retranscrire les entretiens et se familiariser avec les nouveaux logiciels ;

- l'organisation du travail: les tâches sont réparties puis chacune en rend compte aux autres membres et l'analyse est réalisée en commun en classe ainsi que la rédaction ;

- une réflexion : cela se traduit par un état des lieux et une approche critique sur la non-implication ou le désintérêt perçu de la société sur la question du handicap.

Aux premières séances, le groupe témoigne d'une approche négative du handicap dans son approche de l'objet et la question de recherche. Il est question de l'inévitable image négative que la personne affublée d'un stigmate (Goffman, 1963) porte sur elle-même et que les autres portent sur elle. À l'issue de ce travail, le groupe retient une image plus positive de la personne handicapée illustrée par le sujet rencontré. Marie nous est présentée comme une battante. Son discours n'est ni négatif, ni engagé. C'est un discours positif qui est choisi (cf. Encadré 3).

Marie a donc énormément évolué dans le domaine de la natation, mais aussi socialement grâce à la force immense qu'elle puise dans cette pratique sportive. Elle l'explique elle-même ainsi : « il y a donc une évolution dans le sens où les choses que je pensais être impossibles deviennent alors possibles »

Encadré 3. Extrait rapport de TPE, p. 32

Le discours tenu n'est pas pour autant utopique. La jeune fille fait une description de la réalité qu'elle vit avec ses difficultés, ses joies et ses réussites. Ce discours est bien entendu par le groupe qui s'en fait l'écho. Un rapprochement est né mais le discours tenu dans le rapport reste objectif et lucide. Il s'emploie par ailleurs à être constructif. La rencontre est vécue comme positive, voire comme une chance: "nous avons eu la chance de pouvoir assister le samedi 13 décembre 2014 à l'un de ses entraînements et de faire la connaissance de son maitre- nageur ainsi que de tous ses amis nageurs. » (Rapport TPE, p. 31).

Un engagement. L'engagement se traduit par la volonté de transmettre un discours qui se veut positif sur l'objet et de se faire l'écho des difficultés rencontrées. Il s'agit de reconnaître les bienfaits de la pratique sportive et l'intérêt pour la personne à prendre en compte : «elle a commencé à afficher un sourire lorsqu'elle nous a parlé du sport, ce qui nous confirme bien que c'est un atout, une source

\footnotetext{
${ }^{6}$ Education civique juridique et sociale
} 
majeure de joie et de bien-être dans sa vie» (Rapport, annexes, p. 7). L'engagement se traduit par l'écho d'une situation jugée insatisfaisante, celui des infrastructures non adaptées malgré la prescription légale (cf. Encadré 4).

Cependant 10 ans après la promulgation de cette loi, l'ambition initiale n'a malheureusement pas été concrétisée. Ainsi d'après les chiffres de la délégation ministérielle à l'accessibilité, au 1 décembre 2014 sur le million d'établissements recevant du public que compte la France, la proportion de ces établissements totalement accessibles était de seulement $20 \%$ en ajoutant les établissements en travaux cette proportion se monte à un peu plus de $40 \%$.

\section{Encadré 4. Extrait rapport de TPE}

Il est montré, par ailleurs, dès l'introduction la pertinence de l'objet d'étude en général. Le handicap concerne de nombreuses personnes dénommées « concitoyens » dans le texte, qui appuie la référence à l'appartenance au groupe. L'objet est "rarement abordé » et «demeure largement méconnu». Le groupe met en avant une contradiction : une situation qui intéresse nombre d'entre nous et cependant ignorée, insuffisamment diffusée et méconnue par un grand nombre. Le groupe se place dès lors en porte-parole. Il se questionne sur le regard que porte la personne handicapée sur elle-même (cf. Encadré 5), et il ne néglige pas l'importance de la question du regard porté par la société sur le handicap.

Cependant, alors même que le handicap concerne un nombre important de nos concitoyens, ce sujet est
rarement abordé ; le monde du handicap demeure largement méconnu. Nous avons alors choisi de nous
intéresser, au-delà du regard que la société porte sur le handicap, à celui qu'une personne souffrant d'un
handicap porte sur elle-même et aux moyens dont elle dispose pour améliorer justement ce regard

Encadré 5. Extrait rapport de TPE, introduction, p. 1

Une réflexion. La conclusion traduit une réflexion sur l'objet nourri du travail réalisé. S'il est bien question de «lutter», le ton n'est pas à la révolte mais plutôt à la prise de conscience et à la sensibilisation. L'analyse du discours de Marie relatant sa pratique sportive va en ce sens (cf. Encadré $6)$.

\begin{tabular}{l}
\hline 'analyse de la partie du discours portant uniquement sur la question de la Natation est très intéressante, car \\
elle relève un nouveau vocabulaire fort significatif. Alors que la question du handicap semble ici plus \\
présente (fait intéressant en soi), elle est associée à des images fort positives (Bonheur, Avenir) et des verbes \\
d'action (action par opposition à passivité, ce qui nous laisse dire que Marie vît son handicap et ne le subit \\
pas) : bouger, devenir, s'évader, adorer. Le discours reste toutefois réaliste : il est question d'aide qui permet \\
(verbe permettre). Le Sport apparaît comme une activité qui aide facilement à se sentir bien et à se retrouver \\
dans ce corps partiellement immobilisé, et à l'accepter.
\end{tabular}

Encadré 6. Rapport de TPE, annexes, p. 6

Dans la conclusion, le groupe adopte un discours positif et combatif qui semble ouvrir la réflexion sur une nouvelle approche du handicap, ou tout du moins une sensibilisation.

Un ancrage. Toutefois, nous relevons des ancrages dans l'approche du handicap par le groupe : il appartient à la personne handicapée d'interférer pour imposer une image «acceptable ». Cela laisse à dire que son image n'est pas acceptable du fait de son stigmate. La personne affublée d'un handicap ne répond pas aux normes et codes d'une société. Pour appartenir au groupe, elle se doit de se faire accepter par son attitude (attitude positive comme la pratique d'un sport, comme le discours tenu) pour aider à l'approche de l'autre: "certaines personnes ont avoué que le fait de savoir qu'une personne handicapée pratique une activité sportive favorise l'échange et la bonne image sociale.» (Rapport TPE, p. 17). Il appartient à la personne handicapée d' «améliorer » son image. L'approche choisie fait écho à un questionnement identitaire qui enfermerait le sujet. 
Le titre choisi (les moyens qu'utilise une personne souffrant d'un handicap pour améliorer le regard qu'elle porte sur elle-même et celui qu'elle renvoie) fait référence à la souffrance. Il est question de «personne souffrant d'un handicap ». Nous retrouvons le mot « souffrance» dans le corps du texte et dans les réponses aux questionnaires menés en amont. Le terme « souffrance » tranche avec le discours positif tenu. Il correspond au vocabulaire utilisé par le groupe en début de TPE et n'a pas été revu à la fin du travail et de plus s'impose dans le titre.

Devons-nous y analyser un discours positif du groupe tenu par modélisation avec le discours tenu par le sujet-Marie ? Ce discours positif est-il adopté collectivement et individuellement, voire intimement ? Il semble que la vision de souffrance en lien avec le handicap soit suffisamment ancrée pour s'imposer encore après cette rencontre et ce travail réflexif. Goffman (1975) nomme « contacts mixtes » les interactions «à risque » entre «normaux» et «stigmatisés». L'auteur qualifie d'ambivalente l'attitude du normal vis-à-vis du stigmatisé. Le normal s'efforce de voir le stigmatisé comme un être comme les autres. L'attitude n'en serait donc pas spontanée mais calculée, mesurée, et temporaire car, dans le même temps, le normal perçoit le stigmatisé comme une personne anormale.

\subsubsection{Un travail individuel : l'entretien}

L'outil entretien sert à double titre. Il permet d'interroger le sujet-élève sur l'expérimentation se présentant comme un outil de recueil des données. Et il sert à l'expérimentation elle-même comme outil permettant un retour réflexif sur la propre expérience vécue par le sujet.

L'entretien avec Charlotte intervient aux dernières séances, le groupe finit l'écriture du rapport. L'entretien de Charlotte dure 33 minutes. Charlotte est invitée à parler de son TPE et des questions interlocutives viennent relancer si besoin la conversation. La même grille de lecture que pour le rapport est utilisé (cf. Tableau 2).

$\mathrm{Au}$ cours de l'entretien, nous reprenons le questionnaire préalablement complété par Charlotte et revenons sur certaines réponses ou positionnements. Il est à noter notamment qu'à la question d'évocation spontanée (" au mot handicap, j'associe le mot suivant...»,) Charlotte avait noté en tête le mot «souffrance» que nous retrouvons également dans le rapport de TPE. Elle citait ensuite l'image du fauteuil roulant utilisant le mot « chaise » au lieu de «fauteuil ». Elle citait ensuite la malformation. Elle déclarait ne pas connaître de personnes handicapées.

Nous proposons une lecture par items suivant la grille élaborée : Il est question de relever si un travail associant un vécu avec le handicap, la mise en lien avec des savoirs disciplinaires et l'acquisition et l'appropriation de nouveaux savoirs est susceptible de transformer l'approche de l'objet par l'élève et son comportement.

Un vécu. L'expérience vécue à travers l'expérimentation se traduit en deux temps dans le discours de Charlotte : l'approche du travail par la méthodologie de la recherche d'investigation et la rencontre. Ces deux points sont vécus comme sources de connaissances. Il n'y aurait pas eu la rencontre avec Marie sans cette méthode de travail et pas de connaissances à partager sur le handisport sans la rencontre avec Marie. Sans l'expérimentation, il n'y aurait tout simplement pas eu de travail collectif sur l'objet du handicap. Nous relevons l'évolution entre un objet qui n'aurait peut-être pas effleuré le groupe et le discours tenu dans le rapport écrit et par Charlotte où le groupe se dit «très attaché » à la question du handicap.

Un savoir. Charlotte se positionne par opposition à ceux qui ne savent pas mais rappelle qu'il y a peu elle était de ces personnes. Pour Charlotte, la méconnaissance conduit à l'ignorance. L'ignorance dans le sens de ne pas savoir mais également dans le sens de ne pas prêter attention à la question, voire l'indifférence. Charlotte ne se présente pas pour autant comme experte. Il est souvent fait dans son discours des transferts sur ceux qui sont dans l'ignorance. Elle place la-Charlotte-d'avant-le-TPE au rang des personnes ignorants sur la question du handicap. Elle ne place pas pour autant la-Charlottedu-TPE au rang des personnes qui savent. 
Charlotte dit ne pas avoir dans son entourage de personnes handicapées mais à présent elle connaît Marie. Elle a approché les difficultés qui peuvent se rencontrer au quotidien, elle a été témoin des bienfaits de la natation pour Marie, activité qui la transforme "physiquement, psychiquement et socialement ». Son attitude avant le travail de TPE qu'elle décrit comme de l'indifférence tend vers une attitude projetée d'engagement par l'action. Elle loue l'importance de l'information sur le handicap : l'information auprès du corps enseignant (jury) à qui elle relègue la responsabilité d'informer; l'information auprès des proches pour une compréhension de son travail et son nouvel intérêt pour la question du handicap.

Charlotte : (...) je dis pas toutes les semaines d'avoir une séance, mais qu'on sache vraiment ce que c'est. Et qu'on puisse du coup être plus ouvert à quelqu'un qui est, face à quelqu'un qui a un handicap et leur montrer que, je sais comment dire, mais eux [le corps enseignant] aussi sont un peu réticents et ils ne se doutent pas que le sport ça peut les aider. Donc avec le TPE on veut leur montrer que grâce au sport..., eux après notre TPE, ils seront peut-être plus ouverts aussi.... Que çà change un peu leur regard. Et qu'ils se disent eh bein qu'ils sont capables de faire du sport comme nous. Ils peuvent faire de la natation, du foot, n'importe quel sport. Tous les sports sont adaptables à chaque personne.

\section{Encadré 7. Entretien Charlotte, 89ème prise de parole}

Charlotte : (...) je montrerais mon TPE, à ma famille par exemple je montrerais, car quand je leur dis « oui je pars faire un entretien », ils me disent «bien, Ok, ouais », «c'est bien », « C'est bien ton entretien ? », « oui, oui ». Ils (??). Alors que si on fait par exemple un petit film ou que je leur montre par exemple ou que je leur parle, pas forcément en 20 minutes mais je leur fais juste 5 minutes histoire de leur dire bien le sport vraiment ça les aide et tout, et peut-être que ils seront plus ouverts aussi et du coup ... Parce que bon ils sont déjà ouverts avec ça. Ma mère elle a déjà eu dans son travail des personnes à mobi, enfin handicapées. Elle connaissait déjà un petit peu. Mais peut-être que ça l'aidera plus à aller les aborder, à leur parler

Encadré 8. Entretien Charlotte, 98ème prise de parole

Une attitude. Toutefois Charlotte relève que la question est sensible : il n'est pas aisé de parler du handicap que ce soit avec des personnes handicapées ou des personnes valides. Il semble qu'il y ait pour Charlotte une discordance entre le fait que le handicap puisse être visible et que la question soit perçue comme touchant à l'intime. Ce n'est pas un objet plaisant voire douloureux, dérangeant.

Charlotte : (...) parce que, déjà parce qu'on en parle pas souvent. C'est toujours assez, c'est toujours difficile de parler de ce sujet face, enfin avec des gens parce que il y en a qui vont penser euh qui vont être contre, y en a qui vont être direct «on on parle pas de ça », enfin ils vont être vraiment fermés. Et d'autres qui vont être vachement ouverts. Et il y a qui ne sauront pas trop, enfin moi je ne savais pas trop comment, même poser des questions, je ne savais pas trop comment parler du sujet, parce qu'on en parle même pas à l'école. Même pas ... et on ne s'en rend pas compte, tant qu'on est pas concerné je pense, on s'en rend pas compte.

\section{Encadré 9. Entretien Charlotte, 53ème prise de parole}

De plus c'est un objet méconnu. Ce travail lui fait dire que les autres (et peut-être elle-même) doivent changer leur attitude : «il ne faut pas avoir peur de leur parler». L'attitude à adopter par la personne valide est présentée sous la forme d'une prescription, le plus souvent sous la forme négative : "il ne faut pas », " on ne peut pas». Il est davantage fait appel à la morale : «ils sont comme nous, on ne peut pas faire la différence».

Une rencontre. Le ton change lorsque Charlotte témoigne de ce qui l'a visiblement touchée : la rencontre avec Marie, son bien-être lors des séances de natation. Le ton est enthousiasme, même si parfois il est hésitant, si les phrases ne sont pas toujours finies, et que le flux de parole est rapide. 
L'évocation de cette rencontre apparaît très vite dans le discours, juste après avoir exposé la question de recherche et la problématique, et Charlotte y revient régulièrement tout au long de l'entretien.

Charlotte : (...) Et vu qu'on a rencontré une personne, donc, Marie, elle s'appelle, et euh elle nous a dit qu'elle
faisait de la natation. ET çà, la natation, çà l'aide vraiment à ... aller mieux car elle a un problème, donc elle est
paraplégique, mais pas complètement, c'est-à-dire qu'elle peut sentir ses jambes mais elle ne peut pas les bouger.
Donc euh, elle fait la natation et dans la natation vu que l'eau çà la fait flotter elle arrive à se déplacer. Donc elle
travaille surtout avec son dos mais elle se sent ... Enfin dans l'eau on ne la reconnait, on ne se dit pas bein cette
fille elle est... elle se sent plus comme les autres et tout çà. Donc çà l'aide plus facilement à s'intégrer c'est tant
mieux. Puis les gens quand ils savent que on fait du sport, la natation ou n'importe quel sport, ils sont plus
avenants, ils viennent plus à te dire ah mais ils sont peut-être handicapés mais ils font du sport donc ils arrivent
quand même à bouger, ils sont pas cloîtrés et fixés dans leur...

\section{Encadré 10. Entretien Charlotte, 1ère prise de parole}

Charlotte : (...) il [son regard porté sur le handicap] a vraiment changé quand j'ai rencontré Marie... parce qu'elle est super accueillante. Comme elle est... elle a vachement accepté son handicap donc elle en parle librement et elle est vachement ouverte donc ... on lui a dit dès le début parce qu'on savait pas trop comment s'y prendre non plus, c'est difficile de poser des questions à des personnes handic enfin qui ont un handicap. On lui a dit si on, enfin si on dit des choses mal, qu'on les dit mal, il faut, enfin, il faut nous reprendre et tout çà. Et elle a dit « oui pas de soucis ». On a dit qu'on s'excuse aussi parce que des fois on ne sait pas trop comment poser des questions et tout çà. Elle a dit «non mais il n'y a pas de soucis. Dites-moi je corrigerais si il y a un souci. Enfin j'essayerais du mieux que je peux»

Encadré 11. Entretien Charlotte, 29ème prise de parole

\section{La rencontre est également louée comme un apport de la démarche éducative expérimentée.}

Charlotte; (...) Parce que même si on était un peu réticents au début des entretiens, maintenant, personnellement ça m'a, enfin, j'm'dis bon bein c'est bon, j'ai fait déjà çà. Je suis capable de faire d'autres entretiens comme çà. Et heu, çà nous entr ... entraîné, pardon, à faire des compte-rendus, tout çà. Et en plus ça la rendu vachement intéressant. Ça nous donne plus envie de se mettre à fond dans ce truc qu'un truc genre tout tranquille... parce que j'ai des amis qui font l'infini. Alors eux ils ont cherché des trucs sur internet, et qu'est-ce que c'est l'infini... mais c'est un peu ennuyeux. On a l'impression qu'ils s'ennuient. Alors que nous, on a vu des gens. Même si ça demande plus de travail... on a passé plus de temps...c'est plus intéressant. Ça vaut ... c'est pas du temps perdu.

\section{Encadré 12. Entretien Charlotte, 94ème prise de parole}

Un engagement. L'engagement pour Charlotte présente un enjeu social : rompre avec l'isolement, communiquer. L'engagement passe par une sensibilisation, une information. L'information pourrait avoir pour visée de dépasser le sentiment de gêne et de peur pour conduire les personnes valides et les institutions (l'école) à rompre le cloisonnement instauré. Charlotte cite l'organisation du dispositif UPI (devenu ULIS ${ }^{7}$ ) dans son ancien collège qu'elle qualifie de « mise à l'écart ».

Charlotte : Après au collège, vous savez, enfin, au collège c'était bizarre parce que, enfin c'était bizarre... ils avaient fait une classe spéciale, UPI, ça s'appelait, il y avait des enfants qui avaient des troubles de l'ouïe, enfin, aveugle, il n'y avait pas de personnes en fauteuil roulant, soit, c'est soit ils avaient des problèmes de vue ou auditif. Et euh, ils étaient des fois avec nous en cours mais ils étaient vraiment mis à l'écart. Ça veut dire qu'ils mangeaient pas avec nous, donc ça accentuait le fait que on n'allait pas vers eux. Parce que s'ils avaient été dans notre classe, plus souvent et qu'ils mangeaient en même temps que nous, ça aurait, on aurait plus là... parce que ils étaient vraiment mis à l'écart, dans une table, même pas avec tout le monde. Ils étaient dans une salle à part. C'est-à-dire il y avait toutes les salles... au bout du couloir, c'était leur salle. Nous on était au fond, de l'autre côté, vraiment on était séparés.

\footnotetext{
${ }^{7}$ UPI : unités d'intégration pédagogique remplacés par les ULIS : unités localisées pour l'inclusion scolaire. Circulaire $n^{\circ} 2015-129$ du 21-08-2015 (BOEN n³1 du 27-8-2015).
} 
Un ancrage. À l'issue de ce travail et de cette rencontre, des ancrages demeurent et ressortent du discours de Charlotte.

Le sujet témoigne d'une certaine gêne à utiliser le mot handicap ou à donner un nom au stigmate. Les mots ne sont pas prononcés ou restent suspendus, il y a des silences, le ton est baissé. Charlotte parle de «problème » lorsqu'elle évoque le handicap de Marie. Lorsque la gêne s'exprime, le ton employé par la jeune fille est plus enfantin. Elle fait parler les pairs valides et s'inclut dans le groupe pour évoquer la gêne ressentie en utilisant le pronom impersonnel «on». Elle ne manque pas de recourir à des transferts en évoquant la contagion possible lorsqu'elle évoque la survenance d'un accident qui peut arriver à tous.

Dans les exemples donnés, les RS du handicap reste limitée à l'image du «fauteuil roulant» et à «l'accident»-qui plus est l'accident de circulation - comme cause du handicap.

L'attitude allouée à la personne handicapée est pressante. Celle de faire la démarche pour permettre la rencontre. Mais en face, la personne valide se doit d'être réceptive. L'attitude de Marie est jugée positive car elle est «accueillante » mais aussi parce qu'elle a «accepté son handicap » et aide à la rencontre en "parlant librement» de son handicap. Charlotte lui est reconnaissante de l'avoir aidée à franchir ce pas ou en tous les cas de ne pas l'avoir placée dans une situation difficile. Charlotte avoue que la démarche n'allait pas de soi pour elle. Charlotte semble appliquer l'idée défendue par le groupe qu'il appartient à l'être différent de faire cette démarche. Le groupe dévolue à Marie la charge de régir leurs relations. Il en va de sa responsabilité car elle sait que le groupe, lui, ne sait pas. S'il fait mal, il s'en excuse par avance mais ce ne sera pas de sa responsabilité (cf. Encadré 5).

Charlotte : (...) on lui a dit dès le début parce qu'on savait pas trop comment s'y prendre non plus, c'est difficile de poser des questions à des personnes handic enfin qui ont un handicap. On lui a dit si on, enfin si on dit des choses mal, qu'on les dit mal, il faut, enfin, il faut nous reprendre et tout çà. Et elle a dit « oui pas de soucis ». On a dit qu'on s'excuse aussi parce que des fois on ne sait pas trop comment poser des questions et tout çà. Elle a dit «non mais il n'y a pas de soucis. Dites-moi je corrigerais si il y a un souci. Enfin j'essayerais du mieux que je peux »

\section{Encadré 14. Entretien Charlotte, 29ème prise de parole}

Nous retrouvons dans le discours de Charlotte l'idée ancrée dans notre société par des années chargées d'Histoire de la faute portée par l'être différent (la punition du divin pour la faute commise). Charlotte invite à prendre conscience que «ce n'est pas leur faute ».

Charlotte : (...) ça m'a permis de me dire que même si... il ne faut pas avoir un mauvais regard sur eux parce que c'est pas de leur faute

Encadré 15. Entretien Charlotte, 19ème prise de parole

L’image du handicap reste négative : la personne est «bloquée » dans son fauteuil, «isolée, cloitrée, coupée de la société ».

Un retour réflexif. Charlotte déclare que son regard porté sur le handicap a changé avec ce travail de TPE et situe ce changement à sa rencontre avec Marie.

Dans son discours, Charlotte porte un regard réflexif sur son évolution d'appréhender le handicap. Elle reste prudente en utilisant plus souvent le pronom impersonnel «on» que le pronom personnel «je ». Elle est en pleine (re) construction et elle tente, semble-il, de décontextualiser. 
Elle reporte une part de responsabilité à l'école sur le regard négatif porté sur le handicap. Elle souligne un manquement à son rôle d'information: "parce qu'on n'en parle même pas à l'école ». Pour Charlotte le changement passe par l'information et la transmission des connaissances.

Enfin, elle se dit «grandie» de cette expérience. L'adolescent de 16 ans qui se dit grandi est transformé ou en cours de transformation vers l'adulte qu'il sera demain.

\section{Analyse}

Une analyse croisée des données des questionnaires et des discours à travers l'exemple du rapport écrit du groupe choisi et de l'entretien du sujet Charlotte nous permet d'entrevoir les perspectives de la démarche éducative expérimentée mais aussi les obstacles à appréhender.

\subsection{Des représentations du handicap restrictives mais potentiellement transformables}

Les questionnaires soulignent une approche restrictive et différentialiste du handicap peu encline au « vivre ensemble » mais également des éléments comme le vécu et l'information susceptibles d'influer sur les représentations et sur lesquels l'école peut intervenir. La réalité approchée est perçue comme irréversible par le sujet. Dès lors, la transformation ne peut se concevoir que de manière «progressive». La transformation progressive peut se faire sans rupture, par le bais de pratiques nouvelles ou par le biais d'informations.

Nous relevons les trois niveaux de perception à prendre en compte dans toute démarche éducative à visée transformatrice (Legardez, 2001) :

- les évocations spontanées contiennent des termes concrets (fauteuil roulant en tête). La démarche doit prendre en compte ce faible niveau d'abstraction des représentations pour conduire l'élève vers plus d'abstraction et élargir ainsi ses représentations;

- le niveau de centration du sujet sur l'objet handicap est principalement axé sur lui-même et son environnement proche. La démarche doit aider l'élève à extérioriser ses représentations, à prendre de la distance, notamment par la construction d'une problématique pour décontextualiser;

- le niveau de dominance des représentations du handicap est principalement axé sur les ressentis et le vécu. La démarche doit pouvoir prendre en compte ces éléments.

Les sentiments ambivalents exprimés face au handicap nourrissent les quatre sources de l'ancrage développées par Tisseron (2007) que la démarche éducative doit chercher à dépasser :

- les repères narcissiques conduisent à se sentir supérieur et à augmenter l'écart supposé entre le sujet dit différent et le sujet dit ordinaire. Dans les questionnaires la différence est appuyée et justifie les positionnements. La rencontre du groupe avec Marie permet de souligner les ressemblances et de comprendre les différences, ce qui est de nature à réduire l'écart projeté. Charlotte le souligne lorsqu'elle affirme : «ils sont comme nous ».

- mais face à la satisfaction ou la culpabilité de ne pas être dans la situation de l'autre, le sujet dit ordinaire aurait tendance à exprimer de la compassion Ce ressenti ne tend pas à favoriser le «vivre ensemble » : «la compassion ne consiste pas à se sentir supérieur, mais à penser que les autres sont inférieurs, à les plaindre sincèrement » (p. 280). Si le groupe semble avoir dépassé ce stade avec Marie, il ne l'a pas généralisé au handicap et les personnes en situation de handicap en général ; certaines catégories de handicap n'étant pas abordées comme le handicap mental.

- le fantasme de la faute est présent. Cette projection fantasmée développe la pitié et la charité qui ne sont pas non plus des ressentis positifs, dans le sens de favoriser le «vivre ensemble». Si nous le relevons dans les propos de Charlotte après la rencontre et le travail réalisés, c'est pour en rejeter l'idée («ce n'est pas de leur faute »). Nous pouvons y voir ici une progression. 
- le désir de se cacher de sa propre agressivité peut conduire à idéaliser la personne handicapée, notamment par la recherche chez l'autre de l'exploit : Marie participe à des compétitions de haut niveau en natation et cela est souligné par le groupe et par Charlotte au point d'en devenir au fil des séances et des rencontres avec Marie le sujet du TPE. Ce désir peut développer également la capacité à idéaliser ce que le sujet peut réaliser pour le handicap ; Charlotte tient à partager ses nouvelles connaissances et réflexions avec son entourage proche.

Le vécu construit à l'école peut être l'addition de connaissances acquises et appropriées issues de savoirs informels et formels (l'information) et de l'expérience d'une investigation sur le terrain permettant des rencontres avec le handicap (le vécu). L'expérience vécue - qui ne peut être analysée que dans un contexte spécifique - apparaît comme un élément intériorisé qui peut changer la perception. Le vécu, dont l'école serait à l'origine, pourrait être de nature à influer sur les RS au même titre que le vécu dans son entourage proche et le vécu dans sa chair, comme démontré dans des travaux antérieurs (Jodelet, 2003).

\subsection{Une approche éducative à visée transformatrice : perspectives et limites}

L'éducation à est également une éducation sur, par, pour, contre et dans. Il s'agit d'éduquer au handicap pour apprendre sur le handicap par des tâches, actions, moyens et ressources, pour atteindre un ou des objectifs donnés, contre des idées premières à repenser et à réinventer, ce qui nécessite réflexion et esprit critique dans une finalité plus grande d'éducation $a u$ «vivre ensemble ». Sont développés les connaissances, les attitudes, les comportements, l'esprit critique, les échanges, la compréhension, et les actions qui en découlent à travers les transformations de comportement.

L'approche éducative par les éducations à trouve un terrain propice dans le cadre du TPE. Le cadre de l'action éducative invite l'implication et l'engagement de l'élève. Le travail à la fois personnel et collectif demande de problématiser. Il permet l'élaboration de savoirs, la réflexion et le développement d'un esprit critique sur les attitudes, les valeurs, les comportements, les compétences. Au travers des sources d'informations sollicitées, à la fois formelle, non formelle ou informelle, cette approche éducative tend à une prise de conscience et un regard à la fois plus large et plus positif sur le handicap. Approcher l'autre par sa ressemblance et ses capacités, plutôt que par sa différence et ses déficiences stigmatisantes, conduit à un possible « vivre ensemble ».

Le groupe s'efforce d'objectiver la situation de handicap. Il raconte, décrit et explique (à la troisième personne) le quotidien de Marie et sait aussi lui donner la parole pour recueillir son témoignage et ses impressions sur son vécu (à la première personne). Le dispositif de recherche et d'engagement réciproque construit par le groupe permet à Marie de se défaire de toute attitude de victimisation et aux membres du groupe d'entrer avec elle dans un rapport de co-construction de l'objectivation des situations et d'intersubjectivation réciproque.

Le TPE, réalisé pour l'école, à l'école et hors de l'école, a permis une rencontre. Le travail réalisé pour l'école a permis une analyse allant au-delà de ce que pouvait apporter la simple rencontre. Ce travail a permis un retour réflexif, une objectivation de la question de recherche en y relevant sa pertinence sociale, en la situant dans un contexte, et en conduisant à une co réflexion sur des thématiques (comme la socialisation, l'épanouissement personnel, le bien-être, la force contre l'adversité) et une problématisation. En apportant le savoir social à l'école pour l'analyser avec les outils de la recherche scientifique, le groupe a décontextualisé ce savoir pour le re contextualiser avec les apports des savoirs savants, notamment issus de la discipline de référence et des recherches documentaires.

Le rapport écrit nous renseigne principalement sur les savoirs acquis et appropriés sur le handicap par les élèves avec ce travail. Il témoigne de la rencontre avec Marie, de l'appropriation des nouveaux outils, de la créativité des élèves. L'entretien individuel avec Charlotte fait principalement le récit de la rencontre avec Marie. Il est le témoignage d'une expérience vécue. Il nous renseigne également sur les questionnements nés de ce travail, sur la démarche réalisée avec ses difficultés et réticences et sur ses 
satisfactions. Il fait état aussi des connaissances acquises, des réflexions naissantes et des actions projetées.

Les discours témoignent d'un vécu. L'expérience vécue conduit à un retour réflexif et un regard nouveau sur l'objet de recherche. L'expérience vécue à l'extérieur de l'école est devenue une expérience vécue et revécue à l'intérieur de l'école, dans le cadre de la rédaction collective du rapport et des discussions et débats qui sont nées au sein du groupe, mais également sollicitée par les entretiens individuels conduits. Et ce, de manière distancée et objective de nature à déplacer le regard par un vécu construit à l'école.

Le travail élaboré par le groupe laisse entrevoir tout le potentiel d'un travail collectif avec une mise en lien entre une réalité de terrain approchée en direct et les savoirs disciplinaires transmis et (ré)appropriés. Le rapport du groupe et l'entretien de Charlotte nous décrivent une rencontre avec Marie vécue positivement et une prise de conscience citoyenne sur la place du handicap voire une réflexion sur un possible engagement ou rôle à tenir. Mais l'approche du handicap reste fortement ancrée sur les images réductives, du «fauteuil roulant» et de la souffrance associée, et la gêne fasse à l'objet handicap subsiste, même après ce travail où l'élève s'est fortement impliqué. Dès lors, l'approche éducative expérimentée ne peut pas être pensée isolément mais inclus dans l'ensemble d'un parcours éducatif à visée transformatrice et citoyenne.

\section{Conclusion}

La visée transformatrice d'une société inclusive rencontre des obstacles sociétaux et environnementaux que la recherche en éducation peut aider à dépasser. Le handicap n'existe que dans le regard de l'autre en termes de représentations. Tout l'enjeu de la transformation des représentations est la transformation des actions : l'autre accepte de réajuster ses pratiques dans un contexte nouveau qui accueille les différences, par rapport à un système qui nie la possibilité qu'il a de le faire.

Les dimensions relationnelle et réflexive peuvent conduire à un vécu construit à l'école, facteur majeur dans le processus de transformation durable des RS du handicap. Ceci pour permettre de lutter contre toute forme de stigmatisation, d'indifférenciation, de rejet et autres formes de violences à l'encontre de la personne handicapée, qui conduit à des inégalités dans les pratiques (Martinez \& Gadchaux, 2014).

La recherche réalisée identifie des RS du handicap de l'échantillon de population lycéenne ancrées sur une approche essentialiste et différencialiste qui fait obstacle au « vivre ensemble » mais également des éléments susceptibles de transformer ces représentations. L'expérimentation en classe de TPE tend à démontrer qu'une approche éducative s'appuyant sur les dimensions relationnelle et réflexive conduit le sujet-élève-dit-ordinaire à :

- la relation avec l'autre différent, plaçant la rencontre dans l'ordre des possibles ;

- la réflexion sur la position de la société qui l'entoure, voire sur sa propre position, sur l'objet handicap.

La rencontre de trois lycéennes avec une jeune étudiante handicapée a été rendue possible dans le cadre de ce travail scolaire. L'initiation à la méthodologie de la recherche scientifique a permis de dépasser le seul stade de la subjectivité, ce qu'un simple témoignage en classe non suivi de débat constructif - comme nous en avons été le témoin lors d'actions de sensibilisation menées en lycée - ne permet pas pour le sujet-élève. Cette nécessaire distance émanant d'un travail collectif, permet une co construction et une co réflexion dépassant l'impact subjectif et émotionnel du témoignage. La démarche volontaire vers l'autre apporte une véritable dimension relationnelle, qui n'émane pas d'un témoignage imposé en classe pouvant être vécu comme une intrusion voire une violation qui met à mal sa position et son identité. La démarche volontaire dans une posture de chercheur apporte une véritable dimension réflexive, ce qui conduit les lycéennes, sur un objet social dont elles se disent indifférentes 
avant le TPE, à se projeter dans des actions, en dehors de l'école, de transmission des savoirs acquis et des réflexions nées.

Mais si l'expérimentation menée laisse percevoir le champ des possibles, elle ne permet pas à elleseule de dépasser les obstacles.

La complexité du processus des représentations sociales sur un objet social sensible comme le handicap et le lent processus de transformation tendant à dépasser les obstacles, nécessitent une approche éducative que nous nommerons plurielle. La démarche que nous avons expérimentée pourrait être un maillon de cette approche plurielle dans le cadre d'une matrice curriculaire inscrite dans un parcours éducatif à visée transformatrice et citoyenne (Barthes, 2017; Lange, 2011; Lange \& Martinand, 2010 ; Garnier, 2015; Girault, Lange, Fortin-Débart, Delalande, Sommoneaux \& Lebeaume, 2007). Les éducations à permettent de penser une approche plurielle qui serait à la fois pluri, inter et transdisciplinaire et pluridimensionnelle. L'objet handicap pourrait être abordé de différents points de vue par la pluridisciplinarité. Le dialogue et l'échange sur l'objet trouveraient leur place par l'interdisciplinarité. L'objet handicap qui n'appartient pas en propre à une discipline serait travaillé dans le cadre de la transdisciplinarité. Nous abordons dans notre travail la dimension relationnelle et la dimension réflexive. Une éducation plurielle se traduirait par des actions dissociables, complémentaires et interférentes. La place serait donnée aux actions de sensibilisation, à l'information, au débat (Beaufort, Caussidier, Hagège, Hausberger, et al., 2015), à la rencontre, aux stages, à la réflexion, à la construction, au projet, aux savoirs formels, informels et au non-formel (Poizat, 2003)... dans un tout éducation à.

La recherche en éducation peut contribuer à penser une éducation au handicap en s'appuyant sur l'approche éducative des éducations à dont la «force (...) est d'être un outil d'interrogation de la complexité du monde, visant des prises de décision, selon une logique qui met à mal une construction figée du monde » (Floro, 2013, p. 216). Mais cela ne va pas sans interroger la posture de la recherche en éducation pour aborder les éducations à.

\section{Bibliographie}

ABRIC, J.-C. Les représentations sociales : aspects théoriques. Pratiques sociales et représentations. PUF, Paris, 1994.

ALPE, Y., \& LEGARDEZ, A.«Questions socialement vives, enjeux sociaux et didactiques: la création d'un enseignement d'éducation civique juridique et sociale en France ». 13ème congrès international La recherche en éducation au service du développement des sociétés. AMSE, Canada, 2000.

AMEISEN, J. C., HEILBRUNN, B., HERITIER, F., \& HUNYADI, M. L'éternel singulier. Questions autour du handicap. Lectures, Les livres, 2010.

AUDIGIER, F. « Les représentations que les élèves ont de l'histoire et de la géographie: à la recherche des modèles disciplinaires, entre leur définition par l'institution et leur appropriation par les élèves ». ANRT. Université de Lille III, 1993.

AUDIGIER, F., \& TUTIAUX-GUILLON, N. Compétences et contenus: Les curriculums en questions. De Boeck Supérieur, Bruxelles, 2008.

BARDIN, L. L'analyse de contenu. PUF, Paris, 2011.

BARTHES, A. «Quels outils curriculaires pour des « éducations à » vers une citoyenneté politique? » Educations, Vol 171, Open sciences, International sciences and technical edition, ISTE, London, 25-40, 2017.

BARTHES, A., ALPE, Y. "Les "éducations" à, un changement de logique educative? L'exemple de l'education au developpement durableà l'université. » (A. d. Lille, Éd.) Spirale: revue de recherche en éducation, pp. 197-202, 2012.

BARTHES, A., ALPE, Y. «Utiliser les représentations sociales en éducation : Exemple de l'éducation au développement durable ». L'Harmattan, Paris, 2016.

BEAUFORT, S., CAUSSIDIER, C., HAGEGE, H., HAUSBERGER, B., HAUSBERGER T., \& al. « Organiser un débat en classe sur une question scientifique socialement vive : pourquoi et comment ? ». Bulletin de I'APBG, 85-104. $\leq$ hal-01322991>, 2015. 
BEITONE, A., \& LEGARDEZ, A. «Travaux en didactique des sciences économiques, sociales et de gestion ». Publication de I'Université de Provence, 1997.

BELMONT, B., \& VERILLON, A. "Diversité et handicap à l'école ". Quelles pratiques éducatives pour tous. CTNERHI, 2003.

DOISE, W. \& MUGNY, G. Psychologie sociale et développement cognitif. Armand Colin/Masson, Paris, 1997.

EYMARD, C. «Essai de modélisation des liens entre éducation et santé ». Questions vives, 2(5), pp. 13-34. 2004.

FLAMENT, C., \& ROUQUETTE, M. L. Anatomie des idées ordinaires: comment étudier les représentations sociales. A. Colin, Paris. 2003.

FLORO, M. «Education au développement durable, un territoire révélateur. » Education relative à l'environnement, 112013, 2013.

GARNIER, B. "Qu'est-ce qu'une école démocratique? Perspectives historiques en France du XXe au XXle siècle (19002014) ». Educació i Història: revista d'història de l'educació, 43-67. 2015.

GARNIER, C. \& SAUVE, L. « Apport de la théorie des repreprésentations sociales à l'éducation relative à l'environnement - Conditions pour un design de recherche. » Education relative à l'environnement : Regards - Recherches - Réflexions, 1, pp. 65-77, 1999.

GIRAULT, Y., LANGE, J.- M., FORTIN-DEBART, C., DELALANDE, C., SIMONNEAUX, L. \& LEBEAUME, J. " La formation des enseignants dans le cadre de l'éducation à l'environnement pour un développement durable: problèmes didactiques. „ Éducation relative à l'environnement, 6, pp. 119-136. 2007.

GIRAULT, Y. \& SAUVE, L. " L'éducation scientifique, l'éducation à l'environnement et l'éducation pour le développement durable. Croisements, enjeux et mouvances ». Aster, (46), p. 7-30. 2008.

GOFFMAN, E. Stigmate. Les usages sociaux du handicap. Les éditions de minuit, Paris, 1975.

HARMA, K. \& GOMBERT, A. " Effet de la visibilité du handicap et de l'expérience d'intégration sur la représentation sociale du handicap chez de jeunes collégiens. » Travail et formation en éducation, (8). 2011.

HARMA, K., GOMBERT, A. \& ROUSSEY, J. -Y. « Impact of mainstreaming and disability visibility on social representations of disability and otherness held by junior high school pupils.» International Journal of Disability, Development and Education, 60(4), 312-331. 2013.

HARMA, K., GOMBERT, A \& ROUSSEY, J.-Y. « Attitude et distance sociale des élèves non handicapés à l'égard de leurs pairs handicapés. Canadian Journal of Behavioural Science/Revue canadienne des sciences du comportement, " 46(3), 414. 2014.

JODELET, D. Les représentations sociales (éd. 7). PUF, Paris, 2003.

JODELET, D. \& MOSCOVICI, S. Folies et représentations sociales. PUF, Paris, 1989.

LANGE, J.-M. "Penser l'éducation scientifique en termes de contribution à l'éducation au développement durable: l'exemple des sciences de la vie et de la Terre. » Formation et pratiques d'enseignement en questions, 13, 137-156. 2011.

LANGE, J.-M \& BARROCA-PACCARD, M. «Résumé ». Colloque ACFAS, L'émergence des " éducations à": entre continuités et ruptures. Montréal, 2017.

LANGE, J.-M. \& MARTINAND, J.-L. "Éducation au développement durable et éducation scientifique: balises pour un curriculum ». Enjeux contemporains de l'éducation scientifique et technologique. Presses de l'Université, 165-178. Ottawa, 2013.

LEGARDEZ, A. « La didactique des SES; bilan et perspectives ». Publications de I'Université de Provence. Aix-en-Provence, 2001.

LEGARDEZ, A. " L'utilisation de l'analyse des représentations sociales dans une perspective didactique: I'exemple des questions économiques. » Revue des sciences de l'éducation, 30(3), pp. 647-665. 2004.

LEGARDEZ, A., SIMMONEAUX, L. L'école à l'épreuve de l'actualité (Vol. 110). ESF. Issy-les-Moulineaux, 2006.

LEGARDEZ, A., SIMMONEAUX, L. " Développement durable et autres questions d'actualité. » Questions socialement vives dans l'enseigenment et la formation. 2011. 
LO MONACO, G., LHEUREUX, F. \& HALIMI-FALKOWICZ, S. « Test d'Indépendance au Contexte (TIC) et Structure des Représentations Sociales: [Test of Context Independence (TCl) and Structure of Social Representations]. " Swiss Journal of Psychology, 67(2), 119-123. 2008.

MARTINEZ, M.-L. \& GADCHAUX, C. L'appui des approches relationnelles pour favoriser l'accessibilité à l'université. La nouvelle revue de l'adaptation et de la scolarisation, 3, pp. 193-208. 2014.

MOLINER, P., RATEAU, P. \& COHEN-SCALI, V. « Les représentations sociales. Pratique des études de terrain. ॥ Presses universitaires de Rennes. Rennes, 2002.

MORVAN, J. (1988). Représentations des situations de handicap et d'inadaptations chez les éducateurs spécialisés, les assistants de service social et les enseignants spécialisés en formation. CTNERHI-MIRE. Paris, 1988.

MOSCOVICl, S. La psychanalyse, son image et son public : étude sur la représentation sociale de la psychanalyse. PUF, Paris, 1961.

POPLIMONT, C. «Représentations sociales et formation par alternance. » Education permanente, (155), pp. 67-78. 2003.

POIZAT, D. L'éducation non formelle. L'Harmattan, Paris, 2003.

RATEAU, P., \& ROUQUETTE, M. L. Introduction à l'étude des représentations sociales. PUG. Grenoble, 1998.

ROUSSEAU, N. \& BELANGER, S. La pédagogie de l'inclusion scolaire. (Vol. 8). PUQ, 2004.

TISSERON, S. « Questionner notre rapport au handicap ». dans C. GARDOU et D. POIZAT (dir.) Désinsulariser le handicap. (pp. 279-285). ERES, 2007. 